\title{
MENSURAÇÃO DE CAPACIDADES TECNOLÓGICAS INOVADORAS EM EMPRESAS DE ECONOMIAS EMERGENTES: MÉRITOS LIMITAÇÕES E COMPLEMENTARIDADES DE ABORDAGENS EXISTENTES ${ }^{1}$
}

\section{MEASUREMENT OF INNOVATIVE TECHNOLOGICAL CAPABILITY IN EMERGING ECONOMIES INTERPRISES: MERITS, LIMITATIONS AND COMPLEMENTARITIES OF EXISTING APPROACHES}

\author{
Camila S. Loures \\ Escola Brasileira de Administração Pública e de Empresas, \\ da Fundação Getulio Vargas (EBAPE/FGV) \\ E-mail: cloures@fgvmail.br \\ Paulo N. Figueiredo \\ Escola Brasileira de Administração Pública e de Empresas, \\ da Fundação Getulio Vargas (EBAPE/FGV) \\ Praia de Botafogo, 190 sala 510 \\ Rio de Janeiro-RJ/22.250-900 \\ Tel.: + $55212559-5742$ \\ E-mail: paulo.figueiredo@fgv.br
}

\begin{abstract}
RESUMO
Este artigo enfoca os méritos e limitações de duas grandes abordagens existentes sobre mensuração de capacidades tecnológicas inovadoras. A primeira baseia-se em uma perspectiva macro derivada de surveys de inovação; a segunda, uma perspectiva micro, baseia-se em mensuração de tipos e níveis de capacidades para funções tecnológicas específicas em nível de empresas. O artigo concentra-se nesta segunda abordagem e mostra exemplos de suas aplicações empíricas em nível de organizações no Brasil. O artigo identifica uma complementaridade entre essas duas abordagens e conclui que uma combinação entre elas, em estudos de inovação industrial, forneceria uma perspectiva mais realista sobre a realidade industrial, especialmente no Brasil.
\end{abstract}

Palavras-chave: Mensuração, Capacidade tecnológica, Inovação, Industrialização recente, Indicadores de C\&T. 


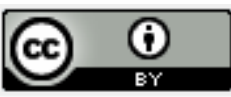

\begin{abstract}
This article focuses the merits and limitations of two existing approaches to the measurement of innovative technological capabilities. The first is based on a macro-level perspective derived from the innovation surveys; the second, a micro-level perspective, is based on the measurement of types and levels of capabilities for specific technological functions at the level of firms. The article concentrates in this second approach and explores examples of its empirical applications at the level of organizations in Brazil. Ten article identifies complementarities between these two approaches and concludes that a combination between them, in studies of industrial innovation, would generate a more realistic perspective on the industrial reality, especially in Brazil.
\end{abstract}

Key-words: Measurement, Technological capability, Innovation, Late industrialization, C\&T indicators.

\title{
1. INTRODUÇÃO
}

A partir do início da década de 1990 houve uma intensificação de estudos baseados em diagnósticos, descrições, análises e propostas relativas ao desenho, re-desenho e implementação de política tecnológica no Brasil orientada para a inovação, desenvolvimento econômico e inserção da economia brasileira no mercado internacional (ver, por exemplo, MARCOVITCH, 1990; MEYER-STAMER, 1995; AMANN; BAER, 1999; MANI, 2001; NICOLSKY, 2001; IEDI, 2002, 2003; ALÁRIO-JUNIOR; OLIVEIRA, 2000; STAUB, 2002). Relativamente ao início da década de 1990, destacam-se os importantes e pioneiros estudos sobre a competitividade de setores industriais da economia brasileira (ver COUTINHO et al., 1993). Não obstante os méritos desses estudos, uma de suas limitações e, particularmente, deste último - é que não foram implementados à base de modelos analíticos e métricas (ou taxonomias) coerentes, ou seja, basearam-se muito pouco, ou quase nada, em modelos analíticos centrados no processo de aprendizagem tecnológica e inovação industrial no contexto de empresas de economias emergentes.

Durante o final da década de 1990, o governo federal, por meio do Ministério da Ciência e Tecnologia, liderou uma meritória iniciativa de sistematizar, de maneira detalhada e exaustiva, os vários elementos relacionados ao sistema de ciência, tecnologia e inovação (CT\&I) considerados necessários ao desenvolvimento nacional, através da edição do documento Ciência, tecnologia e inovação: desafio para a sociedade brasileira - Livro verde 


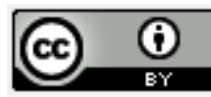

(BRASIL, 2001). Em setembro de 2001, como resultado da Conferência Nacional de CT\&I, foi gerado o Livro branco da ciência, tecnologia e inovação (BRASIL, 2002).

A despeito dos argumentos que advogam a irrelevância ou ausência de estratégia industrial para o desenvolvimento e crescimento da economia brasileira, evidências ao longo da história e, principalmente, durante as últimas décadas, relativas a países que têm alcançado e sustentado taxas significativas de crescimento e de desenvolvimento industrial e econômico, indicam o contrário: tem havido nesses países uma explícita estratégia industrial - com forte ênfase em desenvolvimento tecnológico - que tende a convergir com os objetivos da política macroeconômica. Mais especificamente, a agenda de política macroeconômica, em vez de centrar-se tão somente em aspectos monetários, fiscais e cambiais, também incorpora, com semelhante grau de importância, estratégias de desenvolvimento industrial e tecnológico. Em outras palavras, verifica-se um esforço de convergência entre elementos da política macroeconômica e os da política industrial e tecnológica.

Por isso, é preciso alertar que, ainda que se tenha uma política tecnológica - e industrial - bem desenhada, seus resultados serão medíocres se esta divergir da política macroeconômica. Logo, de um lado, o desenvolvimento tecnológico implica o desenho de políticas governamentais explícitas, como argumentado de maneira clara em Teixeira (2005). De outro, levando-se em conta o princípio básico de gestão segundo o qual se pode gerir com eficácia aquilo que se pode medir e que se pode mudar com êxito aquilo que se pode medir, este artigo concentra-se em duas tarefas: a primeira é clarificar certas definições relativas a estudos empíricos e ao desenho e implementação de estratégias de inovação industrial a partir da perspectiva de aprendizagem tecnológica no contexto de economias emergentes; a segunda é apresentar métricas associadas à operacionalização de tais estratégias e estudos, particularmente no Brasil.

Este artigo enfoca os méritos e limitações de duas grandes abordagens existentes sobre mensuração de capacidades tecnológicas inovadoras. A primeira baseia-se em uma perspectiva macro derivada de surveys de inovação; a segunda, uma perspectiva micro, baseia-se em mensuração de tipos e níveis de capacidades para funções tecnológicas específicas em nível de empresas. O artigo concentra-se nesta segunda abordagem e mostra exemplos de suas aplicações empíricas em nível de organizações no Brasil. O artigo identifica uma complementaridade entre essas duas abordagens e conclui que uma combinação entre elas, em estudos de inovação industrial, forneceria uma perspectiva mais realista sobre a realidade industrial, especialmente no Brasil. 
A partir desta seção introdutória, a Seção 2 fornece conceitos e terminologias referentes à capacidade tecnológica e inovação; a Seção 3 apresenta as origens dos indicadores de C\&T utilizados na mensuração de capacidade inovativa; na Seção 4 são apresentadas algumas limitações dos indicadores de C\&T quando aplicados em contextos de economias de industrialização tardia. Na Seção 5, uma abordagem de mensuração à base de tipos e níveis de capacidades tecnológicas é apresentada, expondo, em seguida, os resultados de uma aplicação prática desta métrica. $\mathrm{O}$ artigo encerra com discussões e recomendações referentes aos mecanismos de mensuração no contexto de economias em desenvolvimento.

\section{CAPACIDADE TECNOLÓGICA E INOVAÇÃO}

Várias são as definições de capacidade tecnológica encontradas na literatura ${ }^{1}$. As mais antigas dizem respeito a uma "atividade inventiva" ou ao esforço criativo sistemático para obter novos conhecimentos em nível da produção (KATZ, 1976). A capacidade tecnológica também inclui as aptidões e os conhecimentos incorporados nos trabalhadores, nas instalações e nos sistemas organizacionais, visando produzir mudanças tanto na produção quanto nas técnicas utilizadas (BELL, 1982; SCOTT-KEMMIS, 1988).

Lall $(1982 ; 1987)$ define capacidade tecnológica como um "esforço tecnológico interno" para dominar novas tecnologias, adaptando-as às condições locais, aperfeiçoando-as e até mesmo exportando-as. Dahlman e Westphal (1982) formularam o conceito de "domínio tecnológico", concretizado através do "esforço tecnológico" para assimilar, adaptar e/ou criar tecnologia, definição análoga à "capacidade tecnológica" de Bell (1982) e Scott-Kemmis (1988). Aprimorando o conceito, Westphal et. al (1984:5) definem capacidade tecnológica como a "aptidão para usar efetivamente o conhecimento tecnológico". Todas essas definições estão associadas aos esforços internos das empresas no sentido de adaptar e aperfeiçoar a tecnologia por elas importada, esforços estes ligados aos aprimoramentos em termos de processos e organização da produção, produtos, equipamentos e projetos técnicos.

A definição de Pack (1987), que entende que a capacidade tecnológica está incorporada em um grupo de indivíduos (por exemplo, gerentes, técnicos e engenheiros), se mostra demasiado limitada e restrita, ignorando o contexto organizacional onde se desenvolvem tais recursos. Para Enos (1991), a capacidade tecnológica envolve o conhecimento técnico (reunido em engenheiros e operadores) e a instituição. Todavia, essa 
definição, assim como a de Pack, sugere que as pessoas são o locus onde residem as capacidades tecnológicas e que as instituições somente as agregam, mas não as incorporam.

Bell e Pavitt $(1993$; 1995) formularam uma definição mais ampla, segundo a qual a capacidade tecnológica incorpora os recursos necessários para gerar e gerir mudanças tecnológicas. Tais recursos se acumulam e se incorporam aos indivíduos (aptidões, conhecimentos e experiência) e aos sistemas organizacionais. Essa definição parece basear-se em outras formuladas anteriormente (KATZ, 1976; LALL, 1982, 1987; DAHLMAN; WESTPHAL, 1982; BELL, 1982; WESTPHAL, 1984; SCOTT-KEMMIS, 1988). A partir da "abordagem baseada nos recursos específicos da firma" (PENROSE, 1959) e valendo-se de evidências empíricas, Bell (1982) faz distinção entre dois tipos de recursos: os necessários para "usar" os sistemas de produção existentes e os necessários para "mudar" os sistemas de produção (que não devem ser tomados como um conjunto distinto de recursos especializados; por serem de natureza difusa, estão amplamente disseminados por toda a organização).

Em outras palavras, a capacidade tecnológica de uma empresa (ou setor industrial) está armazenada, acumulada, em pelo menos, quatro componentes (LALL, 1992; BELL; PAVITT, 1993, 1995; FIGUEIREDO, 2003)²: (a) sistemas técnico-físicos- referem-se à maquinaria e equipamentos, sistemas baseados em tecnologia de informação, software em geral, plantas de manufatura; (b) conhecimento e qualificação das pessoas (também chamada de "capital humano") - referem-se ao conhecimento tácito, às experiências, habilidades de gerentes, engenheiros, técnicos e operadores que são adquiridos ao longo do tempo, abrangendo também sua qualificação formal; (c) sistema organizacional - refere-se ao conhecimento acumulado nas rotinas organizacionais e gerenciais das empresas, nos procedimentos, nas instruções, na documentação, na implementação de técnicas de gestão, nos processos e fluxos de produção de produtos e serviços e nos modos de fazer certas atividades nas organizações; (d) produtos e serviços - referem-se à parte mais visível da capacidade tecnológica, refletindo conhecimento tácito das pessoas e da organização e os seus sistemas físicos e organizacionais; por exemplo, nas atividades de desenho, desenvolvimento, prototipagem, teste, produção e parte da comercialização de produtos e serviços, estão refletidos os outros três componentes da capacidade tecnológica.

Portanto, existe uma relação inseparável entre esses quatro componentes. Capacidade tecnológica, portanto, possui uma natureza difusa e abrangente. Ademais, a capacidade tecnológica é intrínseca ao contexto da firma, região ou país onde é desenvolvida (PENROSE, 1959; DOSI, 1988a; 1988b). ${ }^{3}$ 
Logo, devido à natureza tácita e ampla da tecnologia - e da capacidade tecnológica-, a dimensão organizacional é, de fato, um componente da tecnologia. Por isso, não se faz aqui distinção entre capacidade tecnológica e organizacional - ou entre tecnologia e organização -, já que a última é parte integrante da primeira. Porém, há uma tendência a se negligenciar a dimensão organizacional (e gerencial) da capacidade tecnológica. ${ }^{4}$

\section{ABORDAGENS TRADICIONAIS DE MENSURAÇÃO DA ATIVIDADE INOVADORA À BASE DE INDICADORES DE C\&T}

Oficialmente, foi a Fundação de Ciência Nacional (National Science Foundation NSF) dos Estados Unidos quem iniciou a medição de inovação utilizando a abordagem de resultados: identificação e contagem de inovações tecnológicas comercializáveis (e as características das empresas que as produziram). Aproximadamente na mesma época dos primeiros estudos do NSF, começou o interesse dos países membros da Organização para a Cooperação e o Desenvolvimento Econômico (OCDE) em mensuração da inovação, já que estes consideravam que a performance em inovação era o fator chave para explicar as diferenças entre Estados Unidos e Europa. Dois aspectos foram particularmente mensurados: performance na geração de inovações e performance na difusão de inovações (GODIN, 2002).

Em 1992, o OCDE organizou, em colaboração com Eurostat, um encontro para formatar um questionário padrão e uma lista de questões centrais que permitisse comparação internacional dos levantamentos sobre inovação na Europa. Foi elaborado, então, o Manual de Oslo, que representa a principal fonte internacional de procedimentos e diretrizes para a coleta e interpretação de dados sobre as atividades inovadoras na indústria, com o objetivo de harmonizar metodologias nacionais e coletar informações padronizadas sobre as atividades inovadoras das empresas: o tipo de inovação conduzida, as fontes de conhecimento tecnológico, gastos e atividades relacionadas, os objetivos da empresa, obstáculos e impactos da inovação e das atividades inovadoras (GODIN, 2002).

Os primeiros experimentos baseados no Manual de Oslo foram realizados na Europa em 1993, onde doze países europeus conduziram o primeiro levantamento de atividades inovadoras coordenado, utilizando-o como modelo teórico, conceitual e metodológico, marcando, deste modo, o início da padronização no campo de avaliações sobre inovação (SALAZAR; HOLBROOK, 2004). Seguindo este primeiro ciclo de levantamentos sobre 


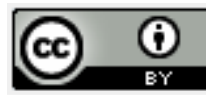

inovação, alguns países em desenvolvimento, sobretudo da América Latina, iniciaram também suas pesquisas baseadas, em grande parte, no Manual de Oslo (SALAZAR; HOLBROOK, 2004).

A primeira revisão (segunda edição) do Manual de Oslo foi desenvolvida em 1996 e encontra-se, neste momento, em sua terceira edição (2005), que foi reorganizada para levar em consideração os principais progressos realizados para o entendimento do processo de inovação e seu impacto na economia (OCDE, 2005).

\section{PRINCIPAIS LIMITAÇÕES DOS INDICADORES CONVENCIONAIS DE C\&T}

Há uma vasta literatura internacional, relativa ao contexto de empresas e países tecnologicamente avançados, que mede capacidade tecnológica de firmas, indústrias e países, à base, por exemplo, de gastos em P\&D (MANSFIELD et al., 1979), qualificações formais (PACK, 1987; JACOBSSON; OSKARSSON, 1995), investimentos em pessoal alocado em laboratórios de P\&D (WORTMAN, 1990) e estatísticas de patentes (PATEL, 1995).

A combinação de estatísticas de patentes e P\&D e outras medidas quantitativas tem sido usada em alguns estudos no Brasil para medir capacidade tecnológica (ver, por exemplo, MACEDO; ALBUQUERQUE, 1999; QUADROS et al., 2001; ANDREASSI; SBRAGIA, 2003; KANNEBLEY, 2003; IBGE, 2006). Tais estudos são extremamente meritórios ao apresentarem uma perspectiva agregada das atividades tecnológicas em empresas no Brasil (FIGUEIREDO, 2004).

Porém, negligenciam o fato de que o desenvolvimento de capacidades tecnológicas e processos de inovação nos países de economias emergentes ocorrem de maneira reversa da apresentada nos países desenvolvidos, havendo, assim, situações em que a utilização dos mesmos indicadores para a medição e identificação de tais atividades em diferentes países e contextos pode levar a informações errôneas e menos relevantes.

Como a base conceitual do Manual de Oslo foi elaborada inicialmente para permitir que pesquisas referentes ao desempenho inovador de um país e suas empresas fossem realizadas nos países desenvolvidos, questões e peculiaridades inerentes às economias emergentes não foram abordadas. Porém, vem sendo utilizado como metodologia de pesquisas também em tais economias. Assim, diversas limitações e críticas surgem quando tais métricas são utilizadas no contexto de países em desenvolvimento. 


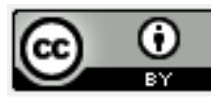

Uma das principais críticas aos levantamentos (surveys) de inovação é a grande ênfase dada aos financiamentos e investimentos em $P \& D$, o que reforça a existência de limitações referentes à utilização do Manual de Oslo como metodologia para os procedimentos de mensuração, já que o manual, embora tenha avançado em relação ao padrão de medida anterior (Manual Frascati), adota como critério-chave a medição de atividades tecnológicas por meio de estatísticas de P\&D (FIGUEIREDO, 2004, 2005).

Muitas vezes, quando se emprega indicadores de $\mathrm{P} \& \mathrm{D}$, tem-se em mente o modelo linear de inovação, através do qual investimentos e recursos aplicados em P\&D levarão automaticamente ao desenvolvimento tecnológico (SALAZAR; HOLBROOK, 2004), ou seja, considera-se que há um caminho quase "infalível" desde as atividades de P\&D até a comercialização de produtos, e que recursos incrementais em P\&D resultam em benefícios incrementais (HOLBROOK, 1997). Porém, inovação não é um processo linear, pelo contrário, os elementos da inovação interagem através dos vários estágios para combinar uma complexa rede de relacionamentos e atividades (ARCHIBUGI; PIANTA, 1996), constituindo muito mais do que simplesmente P\&D.

Outro aspecto sobre as estatísticas de P\&D se refere ao fato de que a incidência de laboratórios de $P \& D$ formalmente organizados nos países de economia emergente é rara, se comparada a alguns setores industriais de países tecnologicamente avançados. Nos países em desenvolvimento, grande parte das atividades tecnológicas inovadoras é conduzida dentro das próprias empresas, em suas unidades organizacionais, nos departamentos de engenharia, de qualidade e manutenção (FIGUEIREDO, 2004, 2005). Assim, a utilização destas estatísticas não contabiliza algumas das principais fontes de acumulação de capacidades nos países emergentes, tais como as pesquisas de engenharia (não P\&D) (LALL, 1994) e a absorção e adoção de conhecimento adquirido externamente (de um competidor, de outra indústria, do governo, de universidades ou outros países) (HOLBROOK, 1997).

A fim de proteger seus produtos e processos da concorrência, as empresas freqüentemente usam patentes para suas inovações, por fornecerem detalhes estatísticos altamente quantificáveis, estarem disponíveis para longo período de tempo (PATEL, 1995) e serem documentos públicos. Por razões legais, patentes são sistematicamente registradas em organismos governamentais (ARCHIBUGI; PIANTA, 1996). Particularmente, a quantidade de patentes registradas nos Estados Unidos é comumente utilizada como critério para medição da atividade tecnológica de determinado país, sendo normalmente aceita como uma medida superior de capacidade tecnológica. Porém, de acordo com Figueiredo (2004; 2005), a 


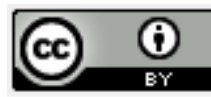

aplicação de tal indicador para economias emergentes pode ser limitante e inadequada, já que tais países não exportam significativamente produtos especializados, com marca própria, para os mercados dos EUA.

Algumas outras desvantagens referentes às patentes podem ser citadas: nem todas as invenções são tecnicamente patenteáveis (por exemplo, no caso de software, que, em geral, estão legalmente protegidos por direito autorais); as empresas, muitas vezes, utilizam outros métodos para proteger suas inovações (por exemplo, segredo industrial, lançamento pioneiro no mercado); o elevado custo para patentear uma invenção; o fato de se as invenções patenteadas realmente se transformam em inovações (ARCHIBUGI; PIANTA, 1996).

Os treinamentos em instituições formais também costumam ser contabilizados para efeitos de mensuração. Porém, utilizados isoladamente, não levam em consideração o treinamento dentro da empresa, em suas operações diárias, através do processo de aprender fazendo e usando, que tipicamente são as principais fontes de desenvolvimento nas empresas de economias emergentes.

De acordo com Figueiredo (2004; 2005), uma das limitações de abordagens baseadas em indicadores convencionais é que não captam as características dos elementos do tecido organizacional, já que é a base organizacional da empresa que influencia o sucesso ou fracasso do engajamento em atividades inovadoras, pois é onde a capacidade tecnológica é desenvolvida, acumulada e sustentada.

Normalmente os enfoques limitados sobre inovação consideram apenas dois tipos de empresas: inovadoras e não-inovadoras, ou seja, focam nas empresas bem-sucedidas (que produziram alguma inovação) em um período de tempo específico, examinando a capacidade tecnológica em um ponto no tempo. Assim, evidenciam uma imagem congelada do processo inovador, onde os desenvolvimentos passados e as capacidades futuras não são questionados nem considerados. Esta abordagem tende a se concentrar nos resultados da inovação, e não apreciar outras variáveis que também fazem parte do processo inovador: em como chegou a tal resultado; as capacidades adquiridas, desenvolvidas e aperfeiçoadas; os tipos de atividades em que a empresa se engajou; o ambiente em que opera.

Assim, estudos à base de indicadores de C\&T tendem a fornecer informações agregadas, voltadas para questões e perspectivas de nível macro, gerando informações capazes de tratar de um grupo mais ou menos uniforme de empresas, sem adentrar nas especificidades de cada uma, nas questões intra-organizacionais, ou então, voltados para 


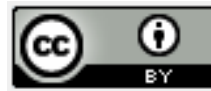

algum aspecto específico (utilizando grupos de indicadores específicos) sem considerar em sua análise aspectos associados mais amplos e igualmente influentes e determinantes.

\subsection{PINTEC: Reflexo de estudos baseados em perspectiva convencional à base de indicadores de C\&T}

\subsubsection{Conceitos e Metodologia da PINTEC}

Para examinar os indicadores convencionais bem como a conformação dos resultados à realidade de países de economias emergentes, este artigo considera a PINTEC como seu objeto de análise. A PINTEC - Pesquisa Industrial de Inovação Tecnológica é realizada pelo Instituto Brasileiro de Geografia e Estatística (IBGE) e tem como objetivo levantar informações sobre distintos aspectos do processo de inovação tecnológica nas empresas brasileiras, que permite a elaboração de indicadores nacionais e regionais, com comparabilidade internacional, fornecendo ferramentas para as empresas definirem suas estratégias e para o desenvolvimento e instrumentação de políticas públicas (IBGE, 2006).

Como referencial conceitual e metodológico, a pesquisa segue as diretrizes definidas pelo Manual de Oslo (OCDE/EUROSTAT de 1997), que se concentra na inovação tecnológica de produtos e processos, e também se apóia na Community Innovation Survey (CIS) - pesquisa aplicada nos países da Comunidade Européia. Sua primeira realização no Brasil ocorreu em 2001, e o segundo levantamento, objeto deste artigo, tem como base o ano de 2003, visando "atualizar os indicadores das atividades de inovação tecnológica nas empresas industriais brasileiras, seguindo as recomendações internacionais” (IBGE, 2006).

Para uma avaliação crítica da PINTEC, recorreu-se à análise do questionário aplicado nas empresas, bem como os resultados da pesquisa. O questionário para a coleta dos dados é composto de 13 capítulos, destinados a coletar informações referentes a características da empresa; produtos e processos tecnologicamente novos ou substancialmente aperfeiçoados; inovação de produto e processo, projetos incompletos e abandonados; atividades inovativas; fontes de financiamento das atividades inovativas; atividades internas de P\&D; impactos das inovações; fontes de informação; cooperação para inovação; apoio do governo; patentes e outros métodos de proteção; problemas e obstáculos à inovação; e outras importantes mudanças estratégicas e organizacionais (IBGE, 2006). 


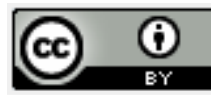

A unidade de análise da pesquisa é a empresa industrial. Foram selecionadas cerca de 11,3 mil empresas, escolhidas dentre empresas industriais ativas, atuantes no território nacional, com o emprego de 10 ou mais pessoas. As informações solicitadas foram respondidas via telefone ou por meio de visita à empresa, através de entrevista com o profissional da empresa com conhecimento das informações requeridas pela pesquisa.

\subsubsection{Méritos e Limitações da PINTEC}

$\mathrm{Na}$ análise do método e dos dados (resultados) da PINTEC, pode-se notar que a pesquisa já incorpora diversas modificações indicadas como necessárias pela literatura, a fim de levar em conta a realidade de economias distintas daquelas dos países desenvolvidos. Por outro lado, alguns pontos ainda precisam ser revistos em uma próxima edição.

O impacto das inovações é medido através da porcentagem das vendas líquidas internas e das exportações (IBGE, 2006). Assim, não captura os esforços inovadores desenvolvidos pelas empresas que não tenham atingido um resultado, ou seja, deixa escapar as medidas tomadas pelas empresas em seu processo de aprendizagem, de acumulação de capacidades, que é um dos principais determinantes de seu desempenho futuro, para tomar apenas as vendas líquidas como medida de sucesso ou fracasso da inovação.

Outro item abordado é referente aos treinamentos realizados e sua importância para a empresa. Porém, são incluídos apenas os programas de treinamento diretamente relacionados às inovações tecnológicas de produto e de processo como, por exemplo, treinamento para a implantação de novas técnicas ou no uso de novas máquinas. Assim, treinamentos que aumentaram as habilidades, o conhecimento tácito das empresas e seus funcionários, que melhoraram a rotina das empresas e das atividades, mas que não tiveram relação necessária com as inovações tecnológicas (resultados) ou não estão relacionados a nenhum mecanismo novo, não são abordados, mesmo com as mudanças incrementais representando um componente essencial no processo de capacitação e desenvolvimento da empresa.

A pesquisa mantém itens referentes a projetos inacabados ou abandonados e sobre as atividades inovativas desempenhadas pelas empresas, procurando medir, em termos monetários, os recursos destinados para as atividades inovadoras. Entretanto, sua análise apenas em termos monetários não é capaz de capturar os processos desenvolvidos por tais atividades, que representa a capacidade da empresa em manter sua posição e até mesmo 
chegar a resultados concretos em um futuro próximo. Além disso, apesar de capturar a presença ou não de projetos incompletos, as possibilidades de identificação de fatores que funcionaram como barreiras e obstáculos e que levaram a sua interrupção ou mesmo abandono é restrita.

Ainda são utilizados muitos indicadores voltados especificamente para as atividades de P\&D tais como o dispêndio total com P\&D; o número de pessoas ocupadas nas atividades de $\mathrm{P} \& \mathrm{D}$ de acordo com a posição (pesquisadores, técnicos e pessoal de suporte), dedicação (exclusiva ou parcial) e nível de qualificação (doutores, mestres, graduados, técnicos de nível médio, outros de suporte); a freqüência (contínua ou ocasional) de tais atividades. Porém, como visto inicialmente, a quantidade de $\mathrm{P} \& \mathrm{D}$ formalmente organizada desenvolvida pelas empresas é pequena nas economias emergentes, onde os resultados aparecem muitas vezes durante suas atividades rotineiras, através de estratégias emergentes.

Constam na pesquisa dados sobre patentes e outros métodos de proteção, o que, nos contextos de economias emergentes, pode acabar gerando dados enganosos, já que a maior parte das empresas não é exportadora de tecnologia, e acabam por privilegiar as inovações tecnológicas efetivas, em detrimento das incrementais. Através dos dados resultantes da pesquisa, apenas 7,4\% das empresas inovadoras utilizam patentes para proteger suas inovações, ou seja, se este indicador for utilizado para indicar o grau de inovação das empresas, a resposta será muito mais pessimista do que a realidade.

Dados sobre o responsável pela inovação (a própria empresa ou outras empresas) e sua localização, o tipo de benefício introduzido, conseguem capturar um pouco dos mecanismos utilizados, bem como as melhorias provenientes de ligações com outras empresas. Além disso, outros indicadores específicos para as fontes de informação utilizadas pelas empresas, seu grau de importância e localização, também informam as redes em que a empresa está inserida e que contribui para seu processo de aprendizagem e capacitação.

Outro item bastante ressaltado na literatura sobre inovação é o fato de que as empresas dificilmente desenvolvem algum projeto inovador completamente sozinhas, uma vez que normalmente se engajam em parcerias. Um destaque para a PINTEC é que engloba este tópico com uma seção específica para tratar das cooperações para inovação, destacando o parceiro, a importância da aliança, a localização e o objeto da cooperação.

Segundo a PINTEC, inovação tecnológica é definida pela introdução no mercado de um produto (bem ou serviço) tecnologicamente novo ou substancialmente aprimorado ou pela introdução na empresa de um processo produtivo tecnologicamente novo ou substancialmente 
aprimorado; tendo sido desenvolvida pela empresa ou adquirida de outra empresa/instituição que a desenvolveu (PINTEC, 2006). Assim, a PINTEC já considera que os mecanismos de difusão fazem parte da realidade das empresas brasileiras como uma das principais fontes de inovação. Dados como a importância de P\&D adquirida externamente pela empresa, aquisição de máquinas e equipamentos, aquisição externa de tecnologia, são levados em consideração como uma importante fonte inovadora para a empresa.

Outro ponto positivo é a inclusão de itens que buscam avaliar as mudanças estratégicas e organizacionais, que também colaboram para o processo de inovação, ou seja, é através de tais mudanças que podem ser estabelecidas diretrizes ou correção de problemas que acabem por fomentar o processo inovador.

O apoio do governo às empresas é outro item importante para as políticas públicas e está contemplado na PINTEC. Assim, pode-se analisar os setores mais (e menos) privilegiados pelo governo, a fim de balancear e ajustar possíveis distorções, além de permitir a análise real da participação efetiva do governo. O resultado mostra que 18,7\% das empresas inovadoras utilizaram o apoio do governo em suas atividades inovativas. Apesar de ser maior do que o resultado anterior (16,9\%), ainda há muito espaço para crescimento.

Diante desta breve exposição, os resultados e a análise da PINTEC sugerem que muitos itens já foram inseridos levando em consideração a peculiaridade de economias emergentes e os aspectos mais amplos da inovação, mas diversos outros tópicos ainda precisam ser repensados. Esse constante questionamento e a busca por adequações e revisões, como o faz o próprio Manual de Oslo, são necessários e saudáveis, já que a inadequação de abordagens, materializadas em questionários, pode levar a resultados enganosos e até mesmo pessimistas, e, conseqüentemente, falhar no seu propósito principal, que é traçar o panorama da inovação de um país, setor ou grupo de empresas, a fim de fornecer informações que sirvam de apoio e subsídio à elaboração de estratégias.

Portanto, apesar dos méritos, muitas limitações ainda podem ser reconhecidas na PINTEC. Algumas delas são facilmente percebidas e podem, e devem, ser prontamente adequadas e discutidas. Outras, entretanto, são mais complexas, difíceis de serem superadas em um tipo de pesquisa como a PINTEC, ou seja, pesquisa agregada, de nível nacional, que busca analisar um conjunto numeroso de questões e envolver os mais variados aspectos que fazem parte do fenômeno da inovação, cobrindo os diversos tipos de indústrias e setores. Para este tipo de pesquisa, a captura de especificidades e de um enfoque com maior nível de profundidade pode se tornar problemática. É neste cenário que se torna necessária a busca de 
complementação em outras metodologias e/ou métricas que dêem conta de capturar dimensões e nuances que indicadores simples não conseguem alcançar.

\section{ABORDAGEM À BASE DE TIPOS E NÍVEIS DE CAPACIDADES TECNOLÓGICAS EM NÍVEL DE EMPRESAS}

\subsection{Modelo para Examinar o Desenvolvimento de Capacidades Tecnológicas}

A fim de examinar as capacidades tecnológicas apresentadas pelas empresas segundo uma métrica alternativa, um modelo para mensuração de capacidades tecnológicas foi desenvolvido por Figueiredo (2001), adaptado de Lall (1992) e Bell e Pavitt (1995), capaz de identificar e medir tipos e níveis de competências tecnológicas em empresas de economias emergentes. Segundo esta abordagem, competência tecnológica representa os recursos aptidões, conhecimentos e experiência, sistemas organizacionais - necessários para gerar e gerir mudanças tecnológicas, ou seja, são as habilidades da empresas para realização de aperfeiçoamentos nas diferentes funções tecnológicas (FIGUEIREDO, 2003).

Através desta taxonomia é possível identificar a natureza e o nível da capacidade tecnológica de empresas ou setores, e não apenas se encontra presente ou não. Faz distinção entre "competências de rotina", definidas como a capacidade para usar/operar certa tecnologia, e "competências inovadoras", que dizem respeito à capacidade para adaptar ou desenvolver novos processos, produtos e sistemas organizacionais, ou seja, são aptidões para modificar tecnologias, conhecimentos, mecanismos organizacionais (FIGUEIREDO, 2005).

De acordo com o modelo (exemplificado pelo Quadro 1), as colunas mostram as capacidades tecnológicas por função (que varia de acordo com a natureza do setor ou empresa pesquisados); e as linhas, por nível de dificuldade (básico, extra-básico, inovativo básico, intermediário, intermediário superior, avançado), que são medidas pelo "tipo de atividade que a empresa é capaz de realizar por si mesma em diferentes intervalos de tempo" (FIGUEIREDO, 2003). Assim, fornece uma base para identificação das competências tecnológicas desde um nível mais básico, onde estão presentes as competências de rotina, até níveis de competências mais avançados, caracterizados pelas competências para inovar.

Apesar de apresentar a capacidade tecnológica em níveis ou "estágios", o modelo não pressupõe a capacitação das empresas na sequiência linear apresentada, ou seja, permite a 


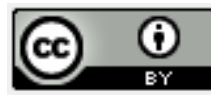

identificação de acumulação de capacidades rotineiras e inovadoras de forma paralela, o que é bastante observado em empresas de economias emergentes (FIGUEIREDO, 2005).

Além da identificação dos níveis de capacidades atingidos pelas empresas, uma aplicação mais sofisticada do modelo permite examinar a velocidade (taxa) de acumulação medida pelo número de anos que uma empresa ou setor industrial leva para alcançar determinado nível de uma função tecnológica específica -, sendo também possível a identificação do tempo em que permaneceu estacionada em determinado nível (FIGUEIREDO, 2005).

Assim, o exame da dinâmica industrial, por meio da identificação dos níveis de acumulação, da preocupação na medição do tempo de acumulação tecnológica (questão ainda bastante negligenciada em estudos empíricos) e a captura da construção e acumulação de capacidades também em níveis intermediários (tidos como pré-condição ao alcance de níveis mais elevados), são alguns dos méritos desta métrica, que pode conduzir a estratégias de inovação focadas e coerentes (FIGUEIREDO, 2005).

\subsection{Aplicação Empírica da Abordagem à Base de Tipos e Níveis de Capacidades}

\section{Tecnológicas}

A aplicação empírica apresentada, resultado de um trabalho desenvolvido por Figueiredo e Marins (2005), baseia-se na abordagem descrita e examina tipos e níveis de capacidades tecnológicas da indústria de tecnologias de informação e comunicação (TICs). São examinados 18 dos principais institutos de pesquisa e desenvolvimento brasileiros, públicos e privados, orientados para o setor de TIC.

No caso do setor de TIC, as capacidades tecnológicas são normalmente mensuradas à base do Capability Maturity Model, que é um modelo gerenciado pelo Software Engineering Institute (SEI) para avaliação da maturidade de processamento de software de uma organização e identificação das práticas requeridas para aumento de tal maturidade. Porém, neste estudo, as capacidades tecnológicas dos institutos de $\mathrm{P} \& \mathrm{D}$ não foram mensuradas unicamente com base nos pré-requisitos e especificações CMM, que mostra-se cada vez mais limitado para mensurar capacidade tecnológica em organizações do setor de TIC (FIGUEIREDO; MARINS, 2005). 


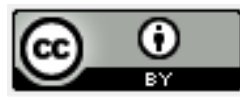

A maioria dos estudos restringe-se a examinar a inovação em serviços apenas em economias tecnologicamente avançadas. De acordo com argumentos convencionais, as atividades de desenho e P\&D permaneceriam em países tecnologicamente avançados, principalmente TIC e suas aplicações, enquanto que países em desenvolvimento estariam confinados às atividades de manufatura. Além disso, sugerem uma deterioração generalizada de capacidade tecnológica inovadora, com as atividades de P\&D cada vez mais concentradas em países industrializados.

Porém, os resultados encontrados nos institutos contrariam os argumentos tradicionais referentes à concentração das inovações em serviços em países desenvolvidos, em oposição aos países em desenvolvimento, fadados e limitados a tarefas menos "criativas". Em termos de desenvolvimento de capacidades tecnológicas, como pode ser observado a partir dos dados apresentados no Quadro 1, há uma diversidade de tipos e níveis de capacidades tecnológicas nos institutos pesquisados, onde nenhum instituto da amostra está confinado a níveis de capacidades de rotina. Todos desenvolveram capacidades até o Nível 3 nas quatro funções examinadas, sendo que um instituto chegou a alcançar o Nível 6 de capacidades, tanto na função Engenharia de Software quanto na de Produtos e Soluções.

Quadro 1. Tipos e níveis de capacidades tecnológicas encontrados nos institutos estudados

\begin{tabular}{|l|c|c|c|c|}
\hline \multirow{2}{*}{$\begin{array}{c}\text { Níveis de } \\
\text { Competência }\end{array}$} & \multicolumn{2}{|c|}{$\begin{array}{c}\text { Atividades de Engenharia e Gestão de Projetos } \\
\text { Engenharia de Software }\end{array}$} & $\begin{array}{c}\text { Produtos e } \\
\text { Soluções }\end{array}$ & $\begin{array}{c}\text { Ferramentas de Projetos } \\
\text { e Processos }\end{array}$ \\
\hline \multicolumn{5}{|c|}{ COMPETÊNCIAS DE ROTINA } \\
\hline Nível 1 (Operações básicas) & $18(100,0 \%)$ & $18(100,0 \%)$ & $18(100,0 \%)$ & $18(100,0 \%)$ \\
\hline Nível 2 (Extra básico) & $18(100,0 \%)$ & $18(100,0 \%)$ & $18(100,0 \%)$ & $18(100,0 \%)$ \\
\hline \multicolumn{5}{|c|}{ COMPETÊNCIAS INOVADORAS } \\
\hline Nível 3 (Inovação básica) & $18(100,0 \%)$ & $18(100,0 \%)$ & $18(100,0 \%)$ & $18(100,0 \%)$ \\
\hline $\begin{array}{l}\text { Nível 4 (Inovação } \\
\text { intermediária) }\end{array}$ & $18(100,0 \%)$ & $14(77,7 \%)$ & $17(94,4 \%)$ & $8(44,4 \%)$ \\
\hline $\begin{array}{l}\text { Nível 5 (Inovação } \\
\text { intermediária superior) }\end{array}$ & $12(66,6 \%)$ & $2(11,1 \%)$ & $7(38,8 \%)$ & $1(5,5 \%)$ \\
\hline Nível 6 (Inovação avançada) & $1(5,5 \%)$ & $0(0,0 \%)$ & $1(5,5 \%)$ & $0(0,0 \%)$ \\
\hline
\end{tabular}

Fonte: Figueiredo e Marins (2005).

Além disso, como observado pelo estudo de Figueiredo e Marins (2005) nos institutos pesquisados, as capacidades ou bases de conhecimento não estão refletidas apenas em seu capital humano e em seus sistemas técnico-físicos (máquinas, softwares, banco de dados). Parte substancial também está acumulada e armazenada nas rotinas, procedimentos, normas e valores das instituições. Ou seja, há que se adotar a perspectiva abrangente de 


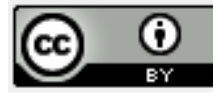

capacidade tecnológica por parte dos gestores, o que implica mudar e gerir bases diversas de conhecimento e distintas especialidades - capacidades ou competências - para realizar atividades ou funções tecnológicas variadas, bem como a necessidade de esforço contínuo e aprofundamento e renovação das capacidades tecnológicas existentes. Novamente, para que se possa gerir de forma eficiente, é necessário, antes, uma identificação e mensuração também eficientes dos níveis já atingidos, bem como a velocidade de evolução.

Outro dado importante verificado através deste estudo refere-se ao fato de que a elaboração de normas e regulamentos internos é o mecanismo de codificação de conhecimento mais incidente nos institutos examinados em todas as fases. Além disso, as certificações ISO e CMM, normalmente tidas como a base de mensuração neste setor, são os mecanismos de menor incidência, evidência que confirma a limitação da certificação CMM como métrica isolada de capacidade tecnológica e ratifica a necessidade de utilização de métricas adicionais para obtenção de dados relevantes e coerentes.

Assim, um dos principais problemas desses argumentos tradicionais, fortemente pessimistas, ainda é o alto grau de generalização sem adequada fundamentação de evidências empíricas. As evidências encontradas a partir desta abordagem sugerem que generalizações, muitas vezes utilizadas, relativas à passividade do sistema de inovação brasileiro nem sempre refletem a realidade industrial do país, sobretudo se confrontadas com fundamentações empíricas que, mensuradas de forma adequada, são capazes de gerar dados e informações relevantes, que contrariam as proposições convencionais.

\section{DISCUSSÕES, CONCLUSÕES E RECOMENDAÇÕES}

Este artigo procurou oferecer uma reflexão crítica sobre a mensuração de capacidade tecnológica no contexto de industrialização recente. Tal reflexão foi realizada à base de uma revisão dos méritos e limitações das abordagens existentes, sintetizados nos Quadros 2 e 3 apresentados a seguir. 


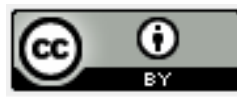

Quadro 2 - Méritos e limitações de abordagens à base de indicadores de C\&T

\section{Méritos}

- Modelo global e genérico, passível de ser aplicado em diversos tipos de empresas/setores/países.

- Permitem a comparabilidade internacional, pela utilização de metodologia padronizada à base de indicadores de C\&T, desenhados para facilitar a captura dos dados por privilegiar, em grande parte das vezes, dados disponíveis em bases/repositórios já existentes nas empresas, tais como arquivos, relatórios contábeis, estoque, fornecedores, descrição de contratos, demonstrações financeiras.

- Normalmente de aplicação periódica e contínua, possibilitam e facilitam a construção de longas séries temporais e a construção de uma base de dados histórica.

- Dados capturados a um baixo custo, em grandes quantidades (ampla amostra), em um período de tempo relativamente curto e baixa taxa de empresas não respondentes.

- Fornecem informações de caráter mais geral, auxiliando a elaboração de políticas para desenvolvimento tecnológico principalmente no nível de países e indústrias, tais como as políticas macroeconômicas, setoriais, cambiais, fiscais, desenvolvimento e fortalecimento do sistema nacional de inovação.

- Procuram fornecer um panorama geral da empresa e seu entorno e do Sistema Nacional/Regional de Inovação (redes de cooperação, agentes existentes, utilização de políticas públicas, fatores externos influenciadores, nível e abrangência das interações, fontes de tecnologia e de informação).

- Algumas versões mais atuais adotam um conceito mais amplo de inovação ( $3^{\mathrm{a}}$ versão do Manual de Oslo e Manual de Bogotá), incluindo elementos tais como as inovações organizacionais, difusão, aquisição externa de tecnologia, melhorias incrementais em produtos ou processos já existentes, inovação em serviços.

- Algumas versões atuais incluem elementos acerca das especificidades de países em desenvolvimento ( $3^{\mathrm{a}}$ versão do Manual de Oslo e Manual de Bogotá): relevância das inovações incrementais; informalidade de atividades de C,T\&I e P\&D; importância e relevância da difusão e da aquisição externa de tecnologia.

- Atualmente, preocupam-se em mensurar o processo e as atividades relacionados à inovação, e não somente os insumos e resultados efetivamente inovadores (no sentido estrito), adotando a abordagem do sujeito, a fim de capturar as atividades inovativas realizadas pelas empresas, incluindo esforços ainda inacabados, não materializados ou mesmo abandonados.

\section{Limitações}

- Tendem a se voltar para a captura de dados e informações mais típicos de empresas da fronteira tecnológica, dando enfoque a atividades e esforços relacionados a níveis mais elevados de desenvolvimento tecnológico (produção de patentes, P\&D formal, exportação, inovações radicais, publicação científica).

- Tendem a abordar os extremos de aspectos que apresentam estágios intermediários, devido ao grande número de questões do tipo sim/não, existe/não existe, utiliza/não utiliza, empresa inovadora/empresa não inovadora, dificultando a possibilidade de identificar aquelas com potencial e capacidade inovativa.

- A maioria não captura evidências acerca das capacidades tecnológicas, sobretudo as de nível mais básico ou intermediário, tendendo a se concentrar naquelas mais avançadas.

- Geram generalizações e informações de forma agregada, sem adentrar nas especificidades e na captura de nuances intra-organizacionais, das diferentes funções tecnológicas, negligenciando a existência de comportamentos e performances distintos nas diferentes atividades praticadas.

- Grande utilização de indicadores de resultado e insumos (produção de patentes, inovações radicais, publicação, gastos com $\mathrm{P} \& \mathrm{D}$, contratação de especialistas), que, isoladamente, não consideram a heterogeneidade das entradas e saídas nem as atividades e esforços contínuos das empresas (aqueles da rotina organizacional, o aprender fazendo e usando, melhorias incrementais em tecnologias já existentes).

- Grande enfoque nas estatísticas de $P \& D$, tais como gastos, pessoal alocado, formalidade, conferindo pouca ou inadequada atenção no $\mathrm{D}$ (desenvolvimento) e nas atividades inovativas que não $\mathrm{P} \& \mathrm{D}$, tais como todas as vertentes de atividades de engenharia, desenho, ferramentaria.

- Tendem a uma abordagem estática e a focar em curtos períodos de tempo (normalmente 2 ou 3 anos), sem procurar obter informações acerca da dinâmica do processo e histórico de desenvolvimento tecnológico, ou seja, não fornecem evidências se, como e a que velocidade as empresas evoluem ao longo do tempo.

- Dificuldade em estabelecer os limites do compromisso (trade off) entre comparabilidade internacional e captura de especificidades. Quando considera somente atividades inovativas típicas, em seu sentido estreito, favorece o primeiro, mas gera resultados pessimistas, negligenciando a maior parte das atividades típicas de empresas de países em desenvolvimento; e se considera inovação em seu sentido amplo, inclui uma gama de atividades não consideradas nos surveys de países desenvolvidos. 
- Dificuldade em contornar o problema da subjetividade das respostas, uma vez que normalmente não são confrontadas/corroboradas com outras fontes de dados, tais como documentação ou arquivos das empresas, o que, pelo tamanho da amostra, demandaria tempo e custos impraticáveis.

\section{Quadro 3 - Méritos e limitações da abordagem à base de tipos e níveis de capacidades}

Méritos

- Permite a identificação de tipos e níveis de capacidades tecnológicas, o que se adequa melhor ao contexto da maioria das empresas dos países em desenvolvimento, já que, por normalmente se caracterizarem pela escassez ou insuficiência de recursos, sobretudo nas etapas iniciais, precisam ser abordadas a partir de uma perspectiva ampla que não se limite a capturar apenas os extremos, ou seja, se a empresa é inovadora ou não é inovadora, mas sim o grau e nível de seu estágio.

- Permite capturar informações tanto de capacidades mais básicas (rotineiras) quanto das intermediárias e inovadoras, não se fixando apenas naquelas capacidades típicas de empresas da fronteira tecnológica, permitindo capturar e mapear os diversos estágios, cuja acumulação não se dá por meio de saltos ou descontinuidades, mas como resultado de esforços que vieram sendo progressivamente implementados e aprofundados até chegarem à condição de geração.

- Exame da dinâmica (velocidade) de evolução das trajetórias de acumulação de capacidades à medida que captura informações relativas a longos períodos de tempo, diferentemente de avaliações baseadas em uma radiografia da empresa em um ponto no tempo, o que tende a ignorar esforços ainda em processo de desenvolvimento e aprofundamento que, mesmo sem produzir efeitos ou resultados concretos, capacitam contínua e progressivamente a empresa. Ao capturar a velocidade de acumulação, permite explorar e contribuir para a dimensão econômica do processo inovativo, por exemplo, fornecendo uma noção mais concreta do tempo para retorno dos investimentos.

- Mais detalhista e aprofundado, uma vez que permite a desagregação em funções tecnológicas e análise intra-organizacional, permitindo adentrar nas especificidades das empresas e em nuances de sua trajetória estagnações, reversões, aumento/redução da taxa de acumulação.

- Possibilidade de avaliar a influência de fatores internos e externos nos tipos e níveis de capacidades tecnológicas acumuladas bem como na velocidade de acumulação.

- Dados confiáveis e robustos, uma vez que a captura se dá, paralelamente, pela observação direta na empresa e pela aplicação de entrevistas, sendo, ainda, complementados com informações obtidas a partir de manuais, documentos e relatórios das empresas e do mercado, restringindo as margens para respostas subjetivas e baseadas em opinião e percepção pessoal dos respondentes.

- Permitem validar/confrontar generalizações tradicionais quanto à inovação em países em desenvolvimento.

- Possibilita a aplicação tanto de análises qualitativas quanto quantitativas, por exemplo, através de testes estatísticos, verificar a existência e o grau de relação e influência de variáveis na trajetória e velocidade de acúmulo de competências tecnológicas.

- Captura outros tipos de atividades que não somente as de P\&D e patentes, tais como atividades baseadas em engenharia (processo, mecânica, eletrônica, elétrica, e engenharia industrial), baseadas em desenho e desenvolvimento, ferramentaria, automação.

\section{Limitações}

- A maioria dos estudos atualmente existentes trata de uma pequena amostra de empresas, normalmente pertencentes a um mesmo setor industrial, dificultando generalizações ou extrapolações para aplicações de caráter mais global e abrangente, fazendo com que a aplicação principal dos resultados existentes seja no nível de empresas ou setores - estratégias corporativas, com limitações para a concretização de elaborações de nível macro, que requer um escopo maior para serem justificadas.

- Por não estar baseada em nenhum manual ou padrão internacionalmente difundido e aplicado, os dados gerados, em sua forma pura, apresentam dificuldades para comparações internacionais de forma direta ou tabulada, pois não geram bases de dados em formato comum àquelas de estratégias padronizadas.

- Tempo e custos elevados: pesquisador em campo, estudo da indústria objeto do estudo, avaliação das funções tecnológicas no âmbito do setor, seleção das funções a serem tratadas, definição das capacidades tecnológicas envolvidas, associação das capacidades a funções específicas, distribuição das capacidades em 
níveis conforme a complexidade, especificação e detalhamento das atividades incluídas em cada um dos níveis e tipos de capacidades, ou seja, não é uma estratégia de aplicação imediata, global ou genérica.

- Dificuldade em examinar em um único estudo os diversos fatores (internos e externos) que influenciam a acumulação de capacidades tecnológicas; normalmente apenas um conjunto deles é selecionado.

- Pode haver dificuldade de isolar/separar completamente as funções tecnológicas, gerando sobreposição das mesmas, já que certas atividades podem estar relacionadas a mais de uma função tecnológica, necessitando do pesquisador esforços adicionais ou mesmo uma decisão baseada em algum parâmetro a ser escolhido.

- Pode necessitar de outros modelos que a complementem para analisar demais fatores influenciadores da trajetória de acumulação de capacidades tecnológicas, tais como o de Figueiredo (2003), que trata da aprendizagem tecnológica, e o de Ariffin (2000), para análise das ligações entre empresas e a forma como contribuem para o acúmulo de capacidades.

É essencial a utilização de abordagens que permitam informar a evolução da empresa ao longo do tempo, ou seja, que representem o processo dinâmico desenvolvido dentro das empresas, expondo sua trajetória tecnológica não somente seu estado em determinado ponto no tempo e, sim, um histórico, que possibilite avaliar sua habilidade de desenvolvimento e sustento de capacidades. Neste sentido, é importante a adoção de perspectivas mais compreensivas sobre inovação. Como visto, há diversos graus de inovação - de básica a complexa, que não são captados pelos estudos à base dos indicadores convencionais. Identificar a progressão por meio dos diferentes estágios de desenvolvimento tecnológico é crucial para entender a dinâmica industrial de economias e regiões em desenvolvimento.

Neste contexto, o artigo apresentou uma abordagem de mensuração, à base de tipos e níveis de capacidades, que permite avaliar, identificar e pontuar, de modo contínuo e à luz de taxonomias coerentes, o nível tecnológico de setores industriais; identificar a maneira e velocidade de acumulação de capacidades tecnológicas ao longo do tempo; identificar os setores com maior potencial, permitindo recomendações de políticas específicas e relevantes para o desenvolvimento de capacidades tecnologias nos diversos setores industriais. Além disso, o modelo permite distinguir as capacidades tecnológicas de rotina das inovadoras, o que é essencial ao processo de desenho de estratégias de inovação industrial, pois são necessários recursos e ações diferentes para cada um dos casos (FIGUEIREDO, 2005).

Isto contribuiria para revisar certas perspectivas limitadas em relação a atividades inovadoras em países em desenvolvimento. Por exemplo, segundo a versão atual do Manual de Oslo (OCDE, 2005), nos países em desenvolvimento "a competitividade é baseada em grande parte na exploração de recursos naturais e mão-de-obra barata, do que em eficiência e produtos diferenciados". Essa perspectiva, por sua vez, não reflete a realidade de certos setores industriais de países em desenvolvimento como é o caso do Brasil. Há diversos estudos que apontam para uma realidade mais positiva e otimista (ver, por exemplo, 
CONSONI, 2004; QUEIROZ; CARVALHO, 2005; FIGUEIREDO; VEDOVELLO, 2005; ARIFFIN; FIGUEIREDO, 2004; FIGUEIREDO; MARINS, 2005). Perspectivas negativas (e equivocadas), podem distorcer decisões sobre investimentos e a elaboração de políticas públicas e estratégias corporativas.

Portanto, a partir da análise realizada neste artigo, pode-se perceber que existem diversos aspectos que merecem destaque nos surveys de inovação conduzidos em países em desenvolvimento. Em primeiro lugar, representam experiências meritórias, altamente importantes e significativas, que vêm se consolidando ao longo do tempo e servindo como fonte e insumo para importantes decisões - empresariais e governamentais. Voltam-se, principalmente, para captura de aspectos e elementos mais amplos e gerais da inovação, a partir de uma perspectiva de níveis meso e macro, de uma lente exterior, de uma grande amostra de empresas, buscando agregar empresas com características semelhantes, avaliar os sistemas nacionais de inovação, as cadeias e fluxos de ligação, os fatores mais influentes da inovação, as características gerais das empresas, sem, contudo, adentrar em suas especificidades, nos aspectos e nuances de sua trajetória ao longo do tempo. Apesar de muitos dos surveys virem sendo conduzidos de forma sistemática, permitindo a construção de séries de dados temporais, ainda são raros aqueles que resgatam dados passados a fim de comporem com os atuais para analisar a evolução das trajetórias ao longo do tempo. Normalmente o que é feito nesse sentido é a exposição de diferença entre aspectos quantitativos gerados, o percentual de modificação/alteração de dados obtidos a partir de um mesmo indicador, sem adentrar e aprofundar nos aspectos qualitativos que este tipo de base de dados é capaz de oferecer. Assim, pode-se dizer que este tipo de estratégia permite comparações internacionais, à medida que se baseia em indicadores padronizados; identificação instantânea de resultados inovadores (produtos e processos inovadores, patentes, publicações), úteis e relevantes do ponto de vista da globalização (onde, muitas vezes, o que realmente importa são os resultados propriamente ditos); uma visão mais completa e abrangente do desenvolvimento tecnológico nacional, à medida que analisa uma amostra significativa de empresas. Entretanto, por carecer de informações mais detalhadas e refinadas, é essencial seu complemento por estratégias que forneçam esta visão mais focada, de longo prazo, detectando nuances e especificidades intraorganizacionais a partir de uma perspectiva dinâmica que aborde o processo de desenvolvimento e acumulação de capacidades tecnológicas e como fatores internos e externos influenciam na direção e taxa desta trajetória. 


\section{REFERÊNCIAS}

ALÁRIO-JUNIOR, D.; OLIVEIRA, N. B. A inovação tecnológica e a indústria nacional. Parcerias Estratégicas, n. 8, p. 45-53, 2000.

AMANN, E.; BAER, W. From technology absorption to technology production: industrial strategy and technological capacity in Brazil's development process. Economia Aplicada, v. 3, n. 1, p. 109-138, 1999.

ANDREASSI, T.; SBRAGIA, R. Fatores Determinantes do Grau de Novatividade das Empresas: um Estudo Utilizando a Técnica de Análise Discriminante. Série de working papers. São Paulo, FEA-USP. No 01/004, 2003. Disponível em: www.ead.fea.usp.br/wpapers.

ARCHIBUGI, D.; PIANTA, M. Measuring technological change through patents and innovation surveys. Technovation, 16(9), 451 - 468, 1996.

ARIFFIN, N. "The Internationalisation of Innovative Capabilities: The Malaysian Electronics Industry", Unpublished Ph.D. Thesis, SPRU, University of Sussex at Brighton. 2000 .

ARIFFIN, N.; FIGUEIREDO, P.N. Internationalization of innovative capabilities: counterevidence from the electronics industry in Malaysia and Brazil. Oxford Development Studies, vol. 32, no 4, 2004.

BELL, M. Technical change in infant industries: a review of the empirical evidence. Brighton: SPRU, University of Sussex, 1982.

; PAVITT, K. Technological accumulation and industrial growth: contrast between developed and developing countries. Industrial and Corporate Change, v. 2, n. 2, p. 157210, 1993.

. The development of technological capabilities. In: UL HAQUE, I.; BELL, M.; DAHLMAN, C; LALL, S.; PAVITT, K. Trade, technology and international competitiveness. Washington, DC: The World Bank, 1995. p. 69-101.

BRASIL. MCT, Ciência, tecnologia e inovação: desafio para a sociedade brasileira Livro verde. Brasília: Ministério da Ciência e Tecnologia, Academia Brasileira de Ciências, 2001.

BRASIL. MCT, Livro branco: ciência, tecnologia e inovação. Brasília: Ministério da Ciência e Tecnologia, 2002.

CONSONI, F. Da tropicalização ao projeto de veículos: um estudo das competências em desenvolvimento de produtos nas montadoras de automóveis no Brasil. Tese de Doutorado. IG/DPCT, UNICAMP, Campinas, 2004 269p.

COUTINHO, L. G.; et al. Estudo da competitividade na indústria brasileira. [S.1.]: IE/Unicamp, Finep, MCT, 1993. 


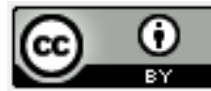

DAHLMAN, C.; WESTPHAL, L. Technological effort in industrial development: an interpretative survey of recent research. In: STEWART, F.; JAMES, J. (Ed.). The economics of new technology in developing countries. London: Frances Pinter, 1982. p. 105-137.

DAHLMAN, C.; FRISCHTAK, C. National Innovation Systems: A Comparative Analysis, ed. Richard Nelson, Oxford University Press, 1993.

DOSI, G. Sources, procedures, and microeconomic effects of innovation. Journal of economic Literature, v. 26, p. 1120-1171, 1988a.

The Nature of the Innovative Process, In: Dosi, G.; Freeman, C.; Nelson, R.;Silverberg, G.; Soete, L. (orgs.), Technical Change and Economic Theory, Londres: Pinter Publishers, 1988b.

ENOS, J. L., The creation of technological capability in developing countries. London: Pinter Publishers, 1991.

FIGUEIREDO P. N. Technological Learning and Competitive Performance. Edward Elgar: Cheltenham, UK \& Northampton, MA, 2001

Learning, capability accumulation and firms differences: evidence from latecomer steel Industrial and Corporate Change, v. 12, n. 3, pp. 607-643, 2003.

Aprendizagem Tecnológica e Inovação Industrial em Economias Emergentes: uma Breve Contribuição para o Desenho e Implementação de Estudos Empíricos e Estratégias no Brasil. Revista Brasileira de Inovação, v.3, n 2, Jul/Dez, p. 323-61, 2004.

Acumulação Tecnológica e Inovação Industrial: conceitos, mensuração e evidência no Brasil. São Paulo em Perspectiva, v.19, n. 1, p. 54-69, 2005.

; VEDOVELLO, C. Firm's Creative Capabilities, the Supporting Innovation System and Globalization in Southern Latin América: A Bleak Technological Outlook or a Myopic Standpoint? Evidence from a Developing Region in Brazil. UNU-INTECH. Discussion Paper Series, 2005.

; MARINS, L. M. Globalização de Competências Tecnológicas Inovadoras no Contexto de industrialização Recente: Evidências de um Amostra de Institutos de Pesquisa e Desenvolvimento (P\&D) em Tecnologias e Informação e Comunicação (TIC) no Brasil. Programa de Pesquisa em Aprendizagem Tecnológica e Inovação Industrial no Brasil, EBAPE-FGV, Relatório Final, Rio de Janeiro, 2005, 295pp.

GODIN, B. The Rise of Innovation Surveys: Measuring a Fuzzy Concept. Project on the History and Sociology of S\&T Statistics, Working Paper, n.16, 2002. Disponível em: $<$ http://www.ost.qc.ca $>$.

HOLBROOK, J. The Use of National Systems of Innovation Models to Develop Indicators of Innovation and Technological Capacity, Third Iberoamerican Workshop on S\&T Indicators. Santiago, Chile: RICyT, 1997. 
IBGE. PINTEC: Dados. 2006. Disponível em: <http://www.pintec.ibge.gov.br>.

IEDI. Clusters ou sistemas locais de produção inovação: identificação, caracterização e medidas de apoio. São Paulo: Iedi, 2002.

. Gargalos da indústria. São Paulo: Iedi, 2003.

JACOBSSON, S.; OSKARSSON, C. Educational statistics as an indicator of technological activity, Research Policy, v. 24, p. 127-36, 1995.

KANNEBLEY, S. Jr. Características das firmas inovadoras no Estado de São Paulo: uma análise empírica a partir da PAEP, Texto para discussão FEA-RP/USP, Série Economia, $\mathrm{n}^{\circ} 40,2003$.

KATZ, J. Importación de tecnología, aprendizaje y industrialización dependiente. México: Fondo de Cultura Económica, 1976.

LALL, S. Technological learning in the Third World: some implications of technology exports. In: STEWART, F.; JAMES, J. (Eds.). The economics of new technology in developing countries. London: Frances Pinter, 1982.

- Learning to industrialise: the acquisition of technological capability by India. London: Macmillan, 1987.

LALL, S. "Technological capabilities and industrialisation", World Development, Vol. 20, No. 2, 165- 86, 1992.

Technological Capabilities. In: Salomon, J. J. et al (orgs.), The Uncertain Quest: Science Technology and Development, Tóquio: UN University Press, 1994.

MACEDO, P.B; ALBUQUERQUE, E.M. P\&D e tamanho da empresa: evidência empírica sobre a indústria brasileira, Revista Estudos Econômicos, v. 29, n.3, p.343-365, 1999.

MANI, S. Government, innovation and technology policy: an analysis of the Brazilian experience during the 1990s. 2001. (UNU/INTECH, Discussion Papers Series, 2001-11.).

MANSFIELD, E.; TEECE, D.; Romeo, A. Overseas research and development by US-based firms, Economica, v. 46, p. 187-196, 1979.

MARCOVITCH, J. Política industrial e tecnológica no Brasil: uma avaliação preliminar. Piensamento Iberoamericano, v. 17, p. 91-117, 1990.

MEYER-STAMER, J. New departures for technology policy in Brazil. Science and Public Policy, v. 22, n. 5, p. 295-304, 1995.

NICOLSKY, R. Inovação tecnológica industrial e desenvolvimento sustentado. Parcerias Estratégicas, v. 13, p. 80-108, 2001. 
OCDE. Proposed Guidelines for Collecting and Interpreting Technological Innovation Data - Oslo Manual. $3^{\mathrm{a}}$ ed. Paris: OCDE, 2005.

PACK, H. Productivity, technology and industrial development: a case study in textiles. New York: Oxford University Press, 1987.

PATEL, P. Localised production of technology for global markets. Cambridge Journal of Economics, v. 19, p. 141-53, 1995.

PENROSE, E. T. The theory of the growth of the firm. Oxford: Basil Blackwell, 1959.

QUEIROZ, S.; CARVALHO, R. Empresas multinacionais e inovação tecnológica no Brasil. São Paulo em Perspectiva, v.19, n. 2, p. 51-99, 2005.

QUADROS, R.; FURTADO, A.; BERNARDES, R.; Franco, E. Technological innovation in Brazilian industry: an assessment based on the São Paulo innovation survey, Technological Forecasting and Social Change, 67 (2-3): 203-219, jun., 2001.

SALAZAR; HOLBROOK, M. A Debate on Innovation Surveys, A conference in honour of Keith Pavitt: "What do we know about innovation?" SPRU, University of Sussex, November, 2003. Science and Public Policy, vol. 31, n. 4, 2004.

SCOTT-KEMMIS, D. Learning and the accumulation of technological capacity in Brazilian pulp and paper firms. World Employment Programme Research, 1988. (Working Paper, 187., p. 2-22).

STAUB, E. Como estimular o investimento e financiar o crescimento. In: SEMINÁRIO "POLÍTICAS DE DESENVOLVIMENTO". Anais... São Paulo: Fiesp/Iedi, 2002.

TEIXEIRA, F.L.C. Desenvolvimento industrial e tecnologia: revisão da literatura e uma proposta de abordagem. Cadernos EBAPE.BR. Edição Especial. Rio de Janeiro: FGV EBAPE, 2005.

WESTPHAL, L. E.; KIM, L.; DAHLMAN, C. J. Reflections of Korea's acquisition of technological capability. Washington, DC: World Bank Research Department, Economics and Research Staff, 1984. (Report DRD77).

WORTMANN, M. Multinationals and the internationalisation of R\&D: new developments in German companies, Research Policy, v. 19, p. 175-83, 1990.

\footnotetext{
${ }^{1}$ Este artigo deriva de parte da dissertação de mestrado em geral empresarial de Camila S. Loures aprovada pela EBAPE/FGV. Uma versão anterior deste artigo foi apreseentada no XXIV Simpósio de Gestão da Inovação Tecnológica, 17-20 Outubro 2006, Gramado, RS. Os autores agradecem aos avaliadores da Revista Produção Online pelas críticas construtivas e comentários encorajadores.

${ }^{2}$ Há uma ampla literatura na qual se busca distinguir entre os termos competências e capacidades tecnológicas. Mais precisamente, na língua inglesa os diferentes termos usados são, por exemplo, capabilities, competence, e competencies. Porém, não é o objetivo deste artigo discutir as diferentes perspectivas, mas apresentar a definição mais ampliada do termo capacidade tecnológica no contexto de economias emergentes. Por isso, daqui em diante será usado apenas o termo capacidade tecnológica. Não obstante, é importante lembrar que o termo
} 
capacitação tecnológica refere-se ao processo de acumulação de capacidades tecnológicas por meio dos vários processos subjacentes de aprendizagem.

${ }^{2}$ Essa perspectiva ampla para capacidade tecnológica também é encontrada na literatura gestão da inovação no contexto de empresas inovadoras de economias industrializadas (ver, por exemplo, Leonard-Barton, 1995).

${ }^{3}$ Logo, é curioso o termo "transferência" de tecnologia. O termo pode transmitir a falsa idéia de que tecnologia pode ser automaticamente transladada de um contexto para outro. No entanto, a real transferência de tecnologia de economias industrializadas para economias emergentes envolve, de um lado, a gestão da aquisição, instalação e da operação da tecnologia importada. De outro, implica assegurar o engajamento da organização recipiente em um contínuo e sistemático de processo de aprendizagem tecnológica. É justamente essa segunda "metade" que tende a ser negligenciada em estratégias de inovação industrial. Isso contribui para explicar o processo irregular de desenvolvimento tecnológico que ocorre em economias emergentes. Para mais detalhes sobre essa perspectiva ver Bell \& Pavitt (1993, 1995) e Bell (1996).

${ }^{4}$ Há uma literatura clássica sobre a relação simbiótica entre tecnologia e organização. Ver, por exemplo, Rosenberg (1976, 1982), Salomon (1984), Pavitt (1985). 